\title{
Title
}

\section{EFFECTS OF PVA-COATED NANOPARTICLES ON HUMAN T HELPER CELL ACTIVITY}

\section{Authors}

Cindy Strehl ${ }^{1,2, *}$, Saskia Schellmann ${ }^{1,2}$, Lionel Maurizi ${ }^{3}$, Margarethe Hofmann-Amtenbrink ${ }^{4}$, Thomas Häupl ${ }^{1}$, Heinrich Hofmann ${ }^{3}$, Frank Buttgereit ${ }^{1,2}$, Timo Gaber ${ }^{1,2}$

\section{Affiliations}

${ }^{1}$ Charité University Hospital, Department of Rheumatology and Clinical Immunology,

10117 Berlin, Germany;

${ }^{2}$ German Rheumatism Research Centre (DRFZ), 10117 Berlin, Germany;

${ }^{3}$ Powder Technology Laboratory, Ecole Polytechnique Federale de Lausanne (EPFL), CH-1015 Lausanne, Switzerland;

${ }^{4}$ MatSearch Consulting Hofmann, CH-1009 Pully-Lausanne, Switzerland;

\section{Corresponding author (Reprint Requests):}

*Dr. Cindy Strehl, Dipl.-Biochem., Department of Rheumatology and Clinical Immunology, Charité University Hospital, Charitéplatz 1, 10117 Berlin, Germany; Phone: ++49 30450 513364, Fax: ++49 30450 513917; e-mail: cindy.strehl@charite.de 


\section{Abstract}

Superparamagnetic iron oxide nanoparticles (SPION) are used as high-sensitive enhancer for magnetic resonance imaging, where they represent a promising tool for early diagnosis of destructive diseases such as rheumatoid arthritis (RA). Since we could demonstrate that professional phagocytes are activated by amino-polyvinyl-alcohol-coated-SPION (a-PVASPION), the study here focuses on the influence of a-PVA-SPION on human T cells activity.

Therefore, primary human CD4+ T cells from RA patients and healthy subjects were treated with varying doses of a-PVA-SPION for $20 \mathrm{~h}$ or $72 \mathrm{~h}$. T cells were then analyzed for apoptosis, cellular energy, expression of the activation marker CD25 and cell proliferation.

Although, we observed that T cells from RA patients are more susceptible to low-dose aPVA-SPION-induced apoptosis than T cells from healthy subjects, in both groups a-PVASPION do not activate CD4+ T cells per se and do not influence mitogen-mediated T cells activation with regard to CD25 expression and cell proliferation. Nevertheless, our results demonstrate that CD4+ T cells from RA patients and healthy subjects differ in their response to mitogen stimulation and oxygen availability.

We conclude from our data, that a-PVA-SPION do neither activate nor significantly influence mitogen-stimulated CD4+T cells activation and have negligible influence on $\mathrm{T}$ cells apoptosis.

\section{Introduction}

Superparamagnetic iron oxide nanoparticles (SPION) have been intensively investigated over the last decades for various in vitro and in vivo biomedical applications ${ }^{1,2}$. Their favorable physicochemical properties and their biocompatibility led to a broad use as contrast 
enhancer for magnetic resonance (MR) imaging ${ }^{3-10}$. Especially with regard to disease detection, progression as well as monitoring of treatment results in the field of destructive disease like rheumatoid arthritis (RA), SPION represent a promising theragnostic tool. RA, a chronic, systemic, inflammatory autoimmune disease, is mainly characterized by the degradation of articular cartilage and subchondral bone ${ }^{11-16}$. There is no cure for RA, treatment strategies can utmost slow down or even stop disease progression but accrued damages are irreversible ${ }^{15,17-19}$. Thus, an early detection, for example with the help of SPION, which are taken up by different immune cells and may enter the inflamed tissue, would be of enormous advantage. However, safety aspects are still under discussion and therefore, adequate and in-depth toxicity studies are necessary prior to routinely in vivo application of nanoparticles. In a previous study in human whole blood we have demonstrated that amino-polyvinyl alcohol (a-PVA) coated SPION do not affect cell survival in general but there was a dose-dependent cell activation as shown by an increased cytokine secretion ${ }^{20}$. Furthermore, human monocytes were affected by a-PVA-SPION since differentiation to and survival of monocyte-derived macrophages (MDM) was increased. In addition, both, monocytes and MDM are able to internalize a-PVA-SPION, as shown in the same study ${ }^{20}$. Others demonstrated that the uptake of nanoparticles as well as their toxic effect strongly depends on the surface properties and thereby with the protein corona formation 21,22 . For example Jurkat cells, a T-lymphoma cell line showed an increased apoptosis and necrosis due to incubation with lauric-acid coated nanoparticles whereas an additional coating of the same nanoparticles with bovine serum albumin reduced these toxic effects ${ }^{22}$. Another group demonstrated that a delivery of nanoparticles to peripheral lymph nodes resulted in enhanced T cell proliferation ${ }^{23}$. 
Thus, since we could already demonstrate that the immune defense, represented by professional phagocytes, is activated by a-PVA-SPION, this report is focused on the influence of a-PVA-SPION on human T cell activation addressing our hypothesis that SPION do not impact cells of the adaptive immune response but professional antigen presenting cells. Furthermore, underlying experiments were performed under normal oxygen availability as well as under pathophysiological hypoxia since this is an important feature of inflamed tissues such as the RA joint.

\section{Material \& Methods}

\section{Materials, reagents \& antibodies}

Amino-polyvinyl alcohol coated superparamagnetic iron oxide nanoparticles (a-PVA-SPION) were synthesized and characterized following a protocol previously described ${ }^{24}$. The mean diameter of the iron oxide core $\left(\mathrm{d}_{\text {core }}\right.$ in $\mathrm{nm}$ ) was measured from the counting of 400 cores via Transmission Electron Microscopy (TEM CM12 from Philips Electron Optics) pictures. The hydrodynamic diameters ( $\mathrm{d}_{\mathrm{H}}$ in $\mathrm{nm}$ ) their polydispersity index (PDI) and Zeta potential (Z pot. in $\mathrm{mV}$ ) of the a-PVA-SPION were measured on a ZetaPals apparatus from Brookhaven (Laborchemie GES MBH). The saturation magnetization (Sat. Mag. In EMU/g) was obtained from freeze-dried powder of naked SPIONs using a superconducting quantum interference device (SQUID). The concentration of PVA (in $\mathrm{mg}_{\mathrm{PVA}} / \mathrm{mg}_{\mathrm{Fe}}$ of nanoparticles) with a thermogravimetric analyses (TGA from Mettler Toledo TGA/SDTA 851e) ${ }^{25}$.

Lectin from Phaseolus vulgaris (PHA-L) and carboxyfluorescein diacetate N-succinimidyl ester (CFSE) were purchased from Sigma-Aldrich Chemie GmbH (Munich, Germany). For flow cytometry, Privigen ${ }^{\circledR}$ human immunoglobulin from CSL Behring GmbH (Marburg, Germany) 
was used. Anti-human CD4-fluorescein isothiocyanate and anti-human CD3-phycoerythrin were obtained from the German Rheumatism Research Center (DRFZ), and anti-human CD25-allophycocyanin was purchased from ImmunoTools GmbH (Friesoythe; Germany).

\section{Cell isolation, -culture $\&$-stimulation}

Venous blood (obtained from RA patients or healthy donors) was collected in heparinized tubes. All patients met the American Rheumatism Association criteria (1987) for RA. The characteristics of RA patients are summarized in supplement 1 . The study protocol was approved by the responsible local administrative body and ethics committee of Charite University Medicine Berlin in accordance with the Declaration of Helsinki. RA patients as well as healthy donors provided written informed consent before enrolment. Human CD4+ T cells (>99\% purity and $>95 \%$ viability) were prepared as described previously ${ }^{26}$. Cells were resuspended in RPMI 1640 (GIBCO) supplemented with 10\% (v/v) heat-inactivated fetal calf serum (Sigma-Aldrich), 100 units $/ \mathrm{ml}$ penicillin $\mathrm{G}, 100 \mu \mathrm{g} / \mathrm{ml}$ streptomycin (both PAA Laboratories $\mathrm{GmbH}$, Cölbe, Germany) and $50 \mu \mathrm{M} \beta$-mercaptoethanol (Sigma-Aldrich).

Cells were stimulated with the mitogen PHA-L $(5 \mu \mathrm{g} / \mathrm{ml})$ and/or a-PVA-SPION $(1 \mu \mathrm{g} / \mathrm{ml}$, $10 \mu \mathrm{g} / \mathrm{ml}$, and $100 \mu \mathrm{g} / \mathrm{ml}$ ) or left untreated for $20 \mathrm{~h}$ (analysis of caspase-3/7-activity (Caspase-Glo 3/7 Assay, Promega GmbH, Mannheim, Germany), intracellular ATP content and CD25 expression) or $72 \mathrm{~h}$ (analysis of proliferation and CD25 expression). Cells were incubated at $37^{\circ} \mathrm{C}$ under either normoxia $\left(18 \% \mathrm{O}_{2} / 5 \% \mathrm{CO}_{2}\right)$ and/or in a hypoxic chamber $\left(5 \% \mathrm{CO}_{2} / \leq 1 \% \mathrm{O}_{2}\right)$ balanced with $\mathrm{N}_{2}$ in humidified incubators (both Binder $\mathrm{GmbH}$, Tuttlingen, Germany). 


\section{Quantification of activation and proliferation}

To measure the extent of proliferation in the PHA-L-activated CD4+ T cells, CFSE was used. The cells were labeled before activation in a $1.5 \mu \mathrm{M}$ CFSE solution for $3.5 \mathrm{~min}$ at room temperature. After $72 \mathrm{~h}$, cells were labeled with anti-CD25 antibody and analyzed by flow cytometry for activation and proliferation status using the MACSQuant analyzer equipped with plate MACSQuant MiniSampler. The acquired data were analyzed using FlowJo 7.6.1 software.

\section{Quantification of intracellular ATP levels}

The samples (50 $\mu \mathrm{L}$ aliquots of each cell suspension) were treated according to manufacturer's instructions (CLS II KIT (Roche Diagnostics GmbH, Mannheim, Germany) and measured against a set of standard dilution rows. Luminescence of all samples was quantified using a luminometer (BD Biosciences, Heidelberg, Germany).

\section{Statistical analysis}

Statistical tests were performed using Graph Pad Prism Software. Data are presented as mean with standard error of the mean (SEM) or box and whiskers, whiskers from minimum to maximum of at least four independent experiments. Outliers were identified with the post-hoc tukey test. Multiple comparisons were analyzed by one way or two way ANOVA as indicated with Bonferroni's multiple comparison post hoc tests for normally distributed data. Differences between normally distributed groups were compared using the Student's $t$ test and in non-normally distributed groups with the Wilcoxon signed rank $t$ test for dependent samples. Probability values of $p<0.05$ were considered to be statistically significant $(* * * p<0.001 ; * * p<0.01 ; * p<0.05)$. 


\section{Results}

\section{a-PVA-SPION characterizations.}

The a-PVA-SPION (Figure 1) have an iron oxide core of around $7 \mathrm{~nm}$ with superparamagnetic properties measured from magnetic saturation at $54 \mathrm{EMU} / \mathrm{g}$. They aggregated in nanoparticles with a mean hydrodynamic diameter of $30 \mathrm{~nm}$ and a positive Zeta potential of $+22 \mathrm{mV}$ at around physiological $\mathrm{pH}$. The mass ratio of PVA per mass of iron on these nanohybrids is 9 .

a-PVA-SPION differentially affect cell count, caspase-3/7-activity, and cellular steady-state ATP level of human CD4+ T cells.

Following the path of our previous investigations ${ }^{20}$, we first focused on the influence of aPVA-SPION on cell survival and cellular energy availability in human CD4+ T cells obtained from RA patients ( $n=11$ ) or healthy donors (HD, $n=12$, figure 2$)$.

When analyzing cell growth/maintenance upon PHA-L treatment we observed for RA patients and healthy donors a significant decrease of cell count (RA: $p<0.01$; HD: $p<0.01$; figure $2 A$ ) and a significant increase in caspase-3/7-mediated apoptosis (RA: $p<0.01$; HD: $\mathrm{p}<0.01$; figure $2 \mathrm{C}$ ) as compared to the untreated control.

Focusing on the influence of a-PVA-SPION we observed a significant decrease in cell count in healthy donors additionally stimulated with PHA-L (all $\mathrm{p}<0.05$; figure $2 \mathrm{~B}$ ) compared to the untreated control. Furthermore, we found a significant increase in caspase-3/7-activity for low-dose a-PVA-SPION $(1 \mu \mathrm{g} / \mathrm{ml})$ treated CD4+ T cells from RA patients and for all a-PVASPION treated, PHA-L stimulated CD4+ T cells from HD (all $p<0.05$; figure 2D) as compared to the untreated control. 
In order to investigate if a-PVA-SPION treatment affects the cellular energy we have analyzed the ATP level of human CD4+ T cells incubated with PHA-L and/or a-PVA-SPION. PHA-L led to a significantly increased steady-state ATP content (compared to untreated control) in both, RA and HD (both $\mathrm{p}<0.05$; figure $2 \mathrm{E}$ ). We further observed significantly reduced steady-state ATP levels in T cells from RA patients at $100 \mu \mathrm{g} / \mathrm{ml}$ a-PVA-SPION ( $p<0.05$; figure $2 \mathrm{C}$ ) and T cells obtained from $\mathrm{HD}$ at $1 \mu \mathrm{g} / \mathrm{ml}$ and $10 \mu \mathrm{g} / \mathrm{ml}$ a-PVA-SPION $(1 \mu \mathrm{g} / \mathrm{ml}: \mathrm{p}<0.05 ; 10 \mu \mathrm{g} / \mathrm{ml}: \mathrm{p}<0.01 ;$ figure $2 \mathrm{~F})$ as compared to the untreated control.

\section{a-PVA-SPION do not activate human CD4+ T cells with regard to CD25 expression.}

To analyze if short term incubation with a-PVA-SPION lead to an increase in T cells activation, we have analyzed the CD25 expression under normoxic and hypoxic conditions. The T cell mitogen PHA-L led to a significantly increased CD25 expression (compared to untreated control) under normoxic and under hypoxic conditions in both, RA ( $n=14, p<0.01$; figure $3 A$ ) and HD ( $n=9, p<0.001$; figure $3 B$ ) T cells. In contrast, a-PVA-SPION at $5 \mu \mathrm{g} / \mathrm{ml}$ did not alter the CD25 expression and there is neither a difference between normoxic and hypoxic conditions, nor between RA patients and HD (figure 3).

\section{a-PVA-SPION do not influence PHA-mediated stimulation of CD25 expression on human CD4+ T cells.}

The question arose if a-PVA-SPION affect an existing T cell activation as it is present in the case of inflammation. Therefore, PHA activated human CD4+ T cells, obtained from RA patients $(n=20)$ or healthy donors $(H D, n=14)$, were treated with a-PVA-SPION under physiological and pathophysiological oxygen conditions. Analysis of CD25 expression revealed that a-PVA-SPION do neither increase nor weaken the CD25 expression in PHA-L- 
activated human T cells (figure 4). In general, the percentage of CD25 positive T cells is slightly higher in RA patients compared to HD.

\section{a-PVA-SPION do not influence PHA-mediated proliferation of human CD4+ T cells}

Moreover, we analyzed the influence of a-PVA-SPION on the proliferation of PHA-activated human CD4+ T cells obtained from RA patients $(n=18)$ or healthy donors (HD, $n=14$, figure 5). As a result, we could not demonstrate any effect of a-PVA-SPION on T cell proliferation in both groups. However, it should be noted that the mean of division index as well as the frequency of divided T cells is slightly increased in HD compared to RA patients (figure 5B $+5 C$ ). We further found a significant diminished proliferation under pathophysiological hypoxia as compared to normoxia in both, RA patients (figure 5B) and HD (figure 5C).

\section{Discussion}

Amino-polyvinyl-alcohol-coated-SPION (a-PVA-SPION) represent a promising tool for early diagnosis of rheumatoid arthritis (RA) and for evaluation of RA treatment when used as highsensitive enhancer for MR imaging taken up by professional phagocytes ${ }^{27}$. Since we could demonstrate that professional phagocytes are activated by a-PVA-SPION in a whole blood system ${ }^{20}$, the study here focused on the influence of a-PVA-SPION on human T cell activity in order to clarify possible effects of a-PVA-SPION on human CD4+ T cells and to target possible long-lasting events in cells which are able to survive for decades in the human body 28. Our data clearly demonstrate that a-PVA-SPION, although slightly and differentially influencing cell count, apoptosis and cellular ATP levels (figure 2), do neither activate nor significantly influence mitogen-stimulated $\mathrm{CD} 4+\mathrm{T}$ cells activation with respect to CD25 expression (figure $3+4$ ) and proliferation (figure 5). 
Several previous data have demonstrated that primarily cells of the innate immune compartment especially professional phagocytes are affected by SPION uptake ${ }^{20,27,29-34}$.

Interestingly, Blank et al. reported that PVA-SPION uptake by human monocyte-derived dendritic cells (MDDC) was decreased by lipopolysaccharide-induced MDDC maturation at higher particle concentrations and that the capacity of MDDC to process antigen was reduced resulting in a diminished $\mathrm{CD} 4+\mathrm{T}$ cells activation ${ }^{29}$. This is in contrast to our findings, since we could not demonstrate any influence of a-PVA-SPION on T cell activation per se. This can at least in part be explained by the small dimension of the a-PVA-SPION, which is around $30 \mathrm{~nm}$ in size ${ }^{20}$. A productive T cell activation is dependent on receptor cross-linking resulting in co-stimulatory signals from other cell surface receptors ${ }^{35}$. Superparamagnetic particles, which are bigger in size $(50 \mathrm{~nm})$, are routinely used to isolate specific types of immune cells from the blood (as it has also been performed in this work here). These particles have been analyzed with regard to cell-specific changes in transcriptional response. As a result it has been demonstrated that there are no significant changes in gene expression pattern as compared to untreated cells ${ }^{36}$. Thus, PVA-SPION may be unable to directly activate human CD4+T cells, but indirect effects should be considered when evaluating SPION effects on human immune cells. Accordingly, the effect of a-PVA-SPION on cells of the innate immune response seems to be acute and transient since data on the effects of a-PVASPION injected, either locally or systemically, in murine experimental arthritis gave an acute inflammatory response which did not aggravate arthritis and did not further activate immune cells or lead to a pro-inflammatory gene expression pattern ${ }^{30}$. 
Finally, we conclude from our data that a-PVA-SPION have no unfavorable influences on CD4+ T cells and are safely to detect joint inflammation by MR imaging with regard to their direct activating potential on CD4+ T cells. 


\section{Figures \& Tables}

Figure 1: Transmission Electronic Microscopy pictures of a-PVA-SPION (with their main physicochemical characteristics)

Figure 2: a-PVA-SPION differentially affect cell count, caspase-3/7-activity, and cellular steady-state ATP level of human CD4+ T cells.

Cells were isolated from human blood samples obtained from patients with RA or HD. Isolated T cells were incubated with PHA-L and ( $n=6)$ / or (RA: $n=11 ; H D: n=12)$ a-PVA-SPION at varying concentrations for 20h. (A, B) Number of cells was quantified; (C, D) caspase-3/7activity was acquired and normalized to cell count and (E, F) steady-state ATP levels were quantified and normalized to cell count. All parameters were normalized to the untreated control (dotted line). Wilcoxon signed rank t test ${ }^{* *} p<0.01, * p<0.05$. RA: rheumatoid arthritis, HD: healthy donors, PHA: Phytohaemagglutinin.

Figure 3: a-PVA-SPION do not activate human CD4+ T cells with regard to CD25 expression. Cells were isolated from human blood samples obtained from (A) patients with RA ( $n=14)$ or (B) healthy donors $(n=9)$ and incubated w/wo PHA or a-PVA-SPION for 20 h under normoxic or hypoxic conditions. Afterwards, cells were analyzed for the surface CD25 expression via flow cytometry. Data are given as box and whiskers; whiskers represent minimum to maximum; one-way ANOVA: ${ }^{* * *} p<0.001,{ }^{* *} p<0.01$. Dots indicate outliers. RA: rheumatoid arthritis, HD: healthy donors, PHA: Phytohaemagglutinin.

Figure 4: a-PVA-SPION do not influence PHA-mediated stimulation of CD25 expression on human CD4+ T cells.

Cells were isolated from human blood samples obtained from (A) patients with RA ( $n=20)$ or (B) healthy donors $(n=14)$ and incubated under influence of PHA and/or a-PVA-SPION at 
varying concentrations for $72 \mathrm{~h}$ under normoxic or hypoxic conditions. Afterwards, cells were analyzed for the surface CD25 expression via flow cytometry. Data are given as box and whiskers; whiskers represent minimum to maximum; one-way ANOVA: ***p $<0.001$. Dots indicate outliers. RA: rheumatoid arthritis, HD: healthy donors, PHA: Phytohaemagglutinin

Figure 5: a-PVA-SPION do not influence PHA-mediated proliferation of human CD4+ T cells. Cell were isolated from human blood samples obtained from patients with RA or healthy donors, labeled with CFSE, stimulated with PHA and incubated for $72 \mathrm{~h}$ under the influence of a-PVA-SPION under normoxic and hypoxic conditions, respectively. Subsequently, cells were analyzed for proliferation by flow cytometry. (A) One example for proliferation analysis (blood obtained from healthy donor) is given here. $(B+C)$ PHA-mediated proliferation of human CD4+T cells obtained from (B) RA patients $(n=18)$ or $(C)$ healthy donors $(n=14)$ by calculation of the division index and the percentage of divided cells. Data are given as box and whiskers; whiskers represent minimum to maximum; one-way ANOVA: ***p<0.001, * p < 0.05. Dots indicate outliers. RA: rheumatoid arthritis, HD: healthy donors, PHA: Phytohaemagglutinin.

\section{Supplement legends:}

Supplement 1: Characteristics of patients with RA at screening visit. Data are given as mean (minimum/maximum) or number (\%); RF, rheumatoid factor; ACPA, anti-citrullinated peptide antibodies; DAS28, disease activity score of 28 joints; ESR, erythrocyte sedimentation rate; CRP, C-reactive protein; DMARDs, disease modifying antirheumatic drugs; NSAIDs, non-steroidal anti-inflammatory drugs; RA, rheumatoid arthritis 


\section{Acknowledgements}

We would like to thank Manuela Jakstadt for outstanding technical assistance, Barbara Szostak and Heide Boeth for sample collection and archiving, and Marie-Gabrielle Beuzelin (EPFL Switzerland) and Usawadee Sakulkhu for their support.

\section{Financial Support}

This work has been supported by the NanoDiaRA project, grant agreement number 228929, funded by the European Seventh Framework Program FP7-NMP-2008-L. 


\section{References}

1. V. I. Shubayev, T. R. Pisanic, 2nd and S. Jin, Advanced drug delivery reviews 61 (6), 467 (2009)

2. K. Maier-Hauff, F. Ulrich, D. Nestler, H. Niehoff, P. Wust, B. Thiesen, H. Orawa, V. Budach and A. Jordan, Journal of neuro-oncology 103 (2), 317 (2011)

3. Y. X. Wang, S. M. Hussain and G. P. Krestin, European radiology 11 (11), 2319 (2001)

4. J. Lodhia, G. Mandarano, N. Ferris, P. Eu and S. Cowell, Biomedical imaging and intervention journal 6 (2), e12 (2010)

5. L. J. Mandarano G, Eu P, Ferris NJ, Davidson R, Cowell SF, Biomedical imaging and intervention journal 6 (2), e13 (2010)

6. D. G. You, G. Saravanakumar, S. Son, H. S. Han, R. Heo, K. Kim, I. C. Kwon, J. Y. Lee and J. H. Park, Carbohydrate polymers 101, 1225 (2014)

7. A. Saraswathy, S. S. Nazeer, N. Nimi, S. Arumugam, S. J. Shenoy and R. S. Jayasree, Carbohydrate polymers 101, 760 (2014)

8. H. Markides, O. Kehoe, R. H. Morris and A. J. El Haj, Stem cell research \& therapy 4 (5), 126 (2013)

9. V. M. Runge, Journal of magnetic resonance imaging : JMRI 12 (2), 205 (2000)

10. J. W. Bulte and D. L. Kraitchman, NMR in biomedicine 17 (7), 484 (2004)

11. M. Feldmann, F. M. Brennan and R. N. Maini, Cell 85 (3), 307 (1996)

12. M. Feldmann, F. M. Brennan, B. M. Foxwell and R. N. Maini, Current directions in autoimmunity 3, 188 (2001)

13. G. S. Firestein, Arthritis and rheumatism 39 (11), 1781 (1996)

14. I. B. McInnes and G. Schett, Nature reviews. Immunology 7 (6), 429 (2007)

15. D. Burger, J. M. Dayer, G. Palmer and C. Gabay, Best practice \& research. Clinical rheumatology 20 (5), 879 (2006)

16. G. Schett, Arthritis research \& therapy 9 Suppl 1, S2 (2007)

17. F. M. Brennan, D. Chantry, A. Jackson, R. Maini and M. Feldmann, Lancet 2 (8657), 244 (1989)

18. M. J. Elliott, R. N. Maini, M. Feldmann, A. Long-Fox, P. Charles, P. Katsikis, F. M. Brennan, J. Walker, H. Bijl, J. Ghrayeb and et al., Arthritis and rheumatism 36 (12), 1681 (1993)

19. P. E. Lipsky, D. M. van der Heijde, E. W. St Clair, D. E. Furst, F. C. Breedveld, J. R. Kalden, J. S. Smolen, M. Weisman, P. Emery, M. Feldmann, G. R. Harriman, R. N. Maini and G. Anti-Tumor Necrosis Factor Trial in Rheumatoid Arthritis with Concomitant Therapy Study, The New England journal of medicine 343 (22), 1594 (2000)

20. C. Strehl, T. Gaber, L. Maurizi, M. Hahne, R. Rauch, P. Hoff, T. Haupl, M. HofmannAmtenbrink, A. R. Poole, H. Hofmann and F. Buttgereit, International journal of nanomedicine 10, 3429 (2015)

21. U. Sakulkhu, M. Mahmoudi, L. Maurizi, G. Coullerez, M. Hofmann-Amtenbrink, M. Vries, M. Motazacker, F. Rezaee and H. Hofmann, Biomaterials Science 3 (2), 265 (2015)

22. J. Zaloga, C. Janko, J. Nowak, J. Matuszak, S. Knaup, D. Eberbeck, R. Tietze, H. Unterweger, R. P. Friedrich, S. Duerr, R. Heimke-Brinck, E. Baum, I. Cicha, F. Dorje, S. Odenbach, S. Lyer, G. Lee and C. Alexiou, International journal of nanomedicine 9, 4847 (2014)

23. L. J. Cruz, P. J. Tacken, I. S. Zeelenberg, M. Srinivas, F. Bonetto, B. Weigelin, C. Eich, I. J. de Vries and C. G. Figdor, Molecular pharmaceutics 11 (12), 4299 (2014)

24. F. Schulze, A. Dienelt, S. Geissler, P. Zaslansky, J. Schoon, K. Henzler, P. Guttmann, A. Gramoun, L. A. Crowe, L. Maurizi, J. P. Vallee, H. Hofmann, G. N. Duda and A. Ode, Small, (2014)

25. L. Maurizi, U. Sakulkhu, L. A. Crowe, V. M. Dao, N. Leclaire, J.-P. Vallee and H. Hofmann, RSC Advances 4 (22), 11142 (2014)

26. R. Tripmacher, T. Gaber, R. Dziurla, T. Haupl, K. Erekul, A. Grutzkau, M. Tschirschmann, A. Scheffold, A. Radbruch, G. R. Burmester and F. Buttgereit, European journal of immunology 38 (6), 1631 (2008) 
27. A. Gramoun, L. A. Crowe, L. Maurizi, W. Wirth, F. Tobalem, K. Grosdemange, G. Coullerez, F. Eckstein, M. I. Koenders, W. B. Van den Berg, H. Hofmann and J. P. Vallee, Arthritis research \& therapy 16 (3), R131 (2014)

28. M. K. MacLeod, J. W. Kappler and P. Marrack, Immunology 130 (1), 10 (2010)

29. F. Blank, P. Gerber, B. Rothen-Rutishauser, U. Sakulkhu, J. Salaklang, K. De Peyer, P. Gehr, L. P. Nicod, H. Hofmann, T. Geiser, A. Petri-Fink and C. Von Garnier, Nanotoxicology 5 (4), 606 (2011)

30. E. A. Vermeij, M. I. Koenders, M. B. Bennink, L. A. Crowe, L. Maurizi, J. P. Vallee, H. Hofmann, W. B. van den Berg, P. L. van Lent and F. A. van de Loo, PloS one 10 (5), e0126687 (2015)

31. K. Schulze, A. Koch, A. Petri-Fink, B. Steitz, S. Kamau, M. Hottiger, M. Hilbe, L. Vaughan, M. Hofmann, H. Hofmann and B. von Rechenberg, Journal of nanoscience and nanotechnology 6 (9-10), 2829 (2006)

32. I. Raynal, P. Prigent, S. Peyramaure, A. Najid, C. Rebuzzi and C. Corot, Investigative radiology 39 (1), 56 (2004)

33. O. Lunov, V. Zablotskii, T. Syrovets, C. Rocker, K. Tron, G. U. Nienhaus and T. Simmet, Biomaterials 32 (2), 547 (2011)

34. S. Naqvi, M. Samim, M. Abdin, F. J. Ahmed, A. Maitra, C. Prashant and A. K. Dinda, International journal of nanomedicine 5, 983 (2010)

35. J. E. Smith-Garvin, G. A. Koretzky and M. S. Jordan, Annual review of immunology 27, 591 (2009)

36. P. A. Lyons, M. Koukoulaki, A. Hatton, K. Doggett, H. B. Woffendin, A. N. Chaudhry and K. G. Smith, BMC genomics 8, 64 (2007) 
Figure 1

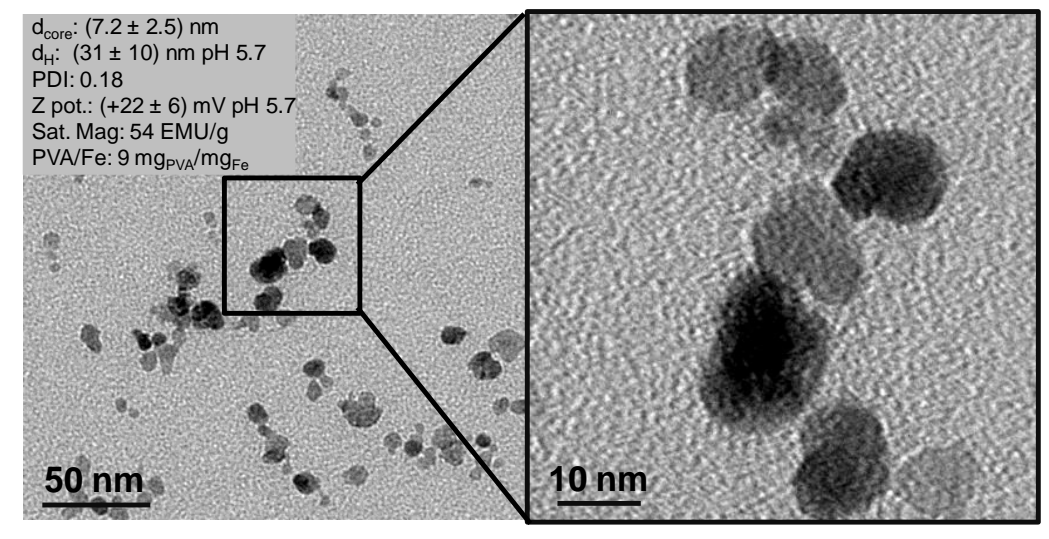


Figure 2

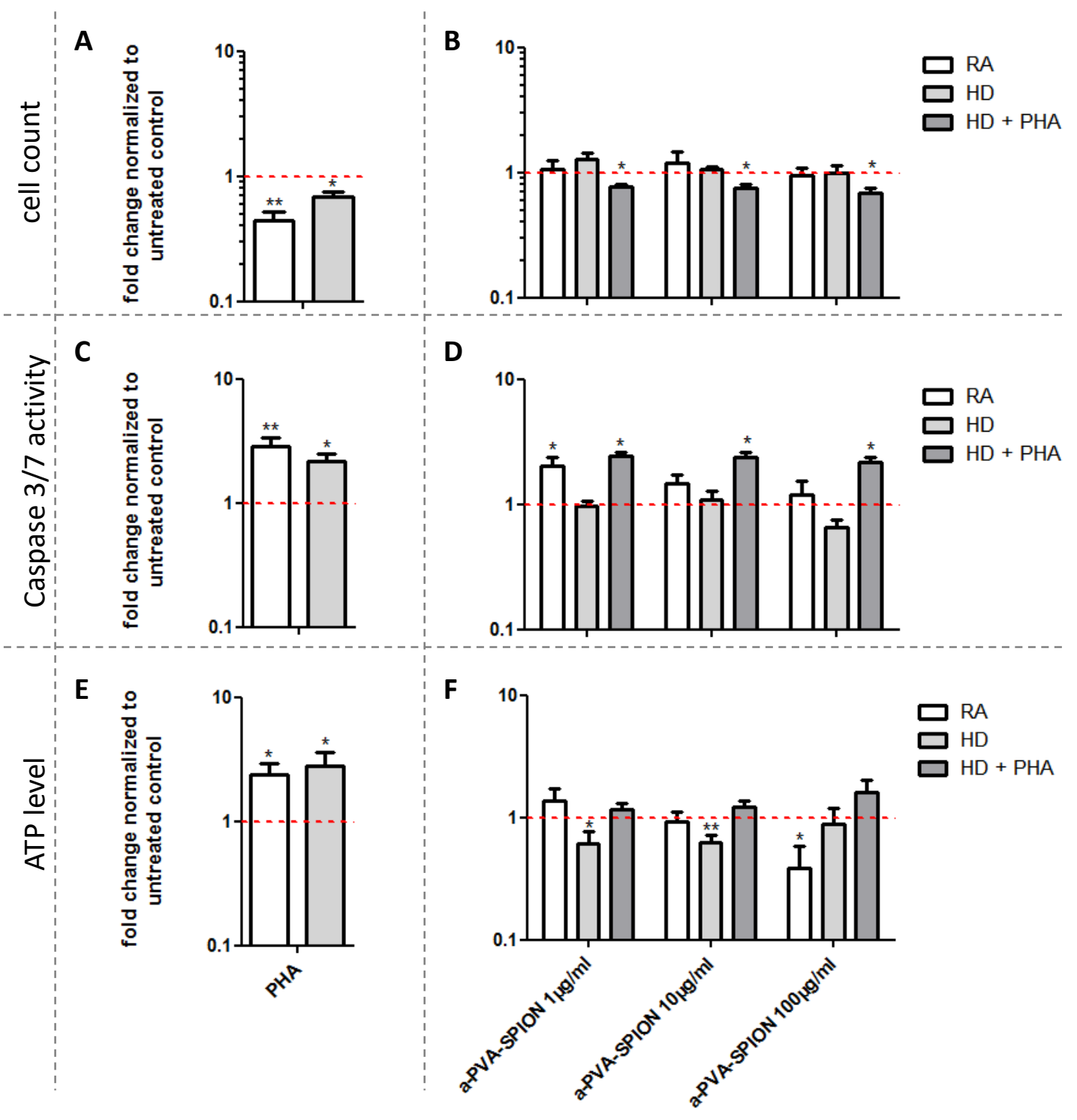


Figure 3
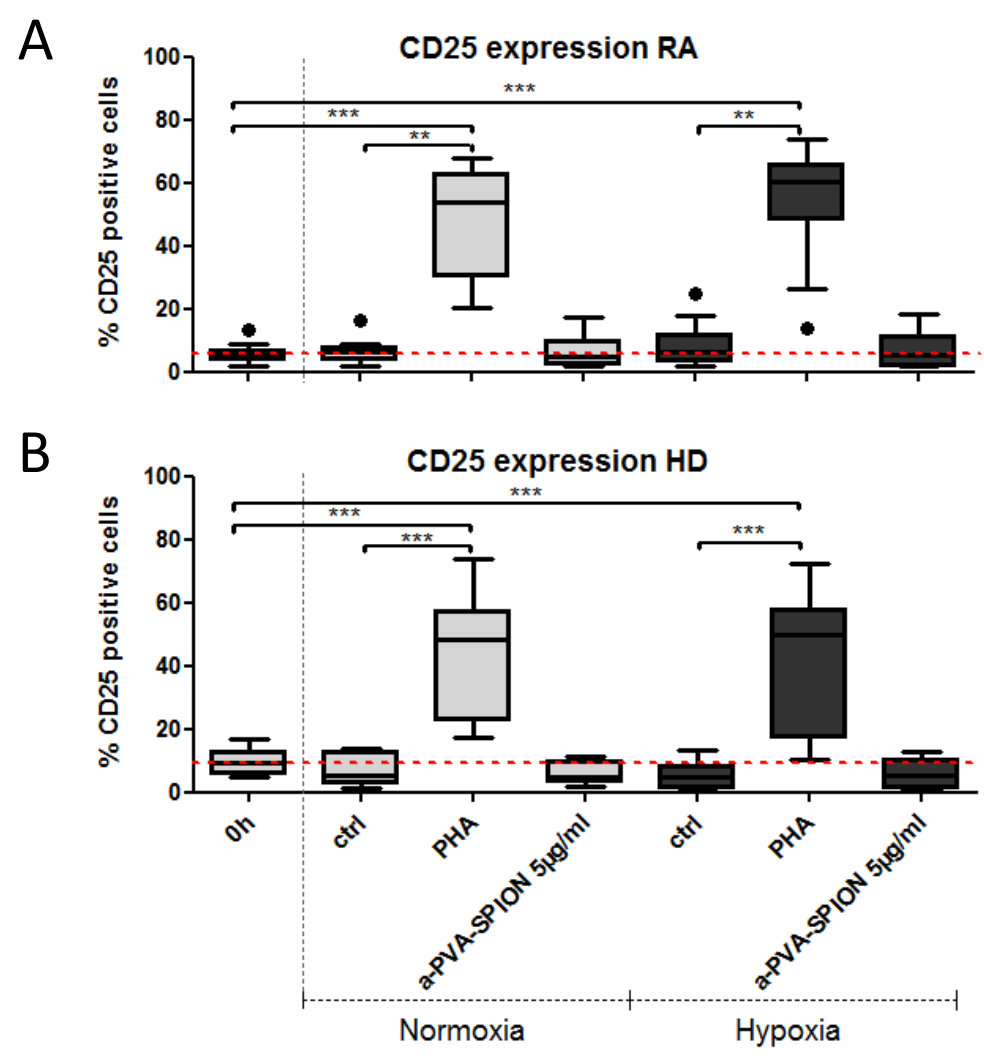
Figure 4
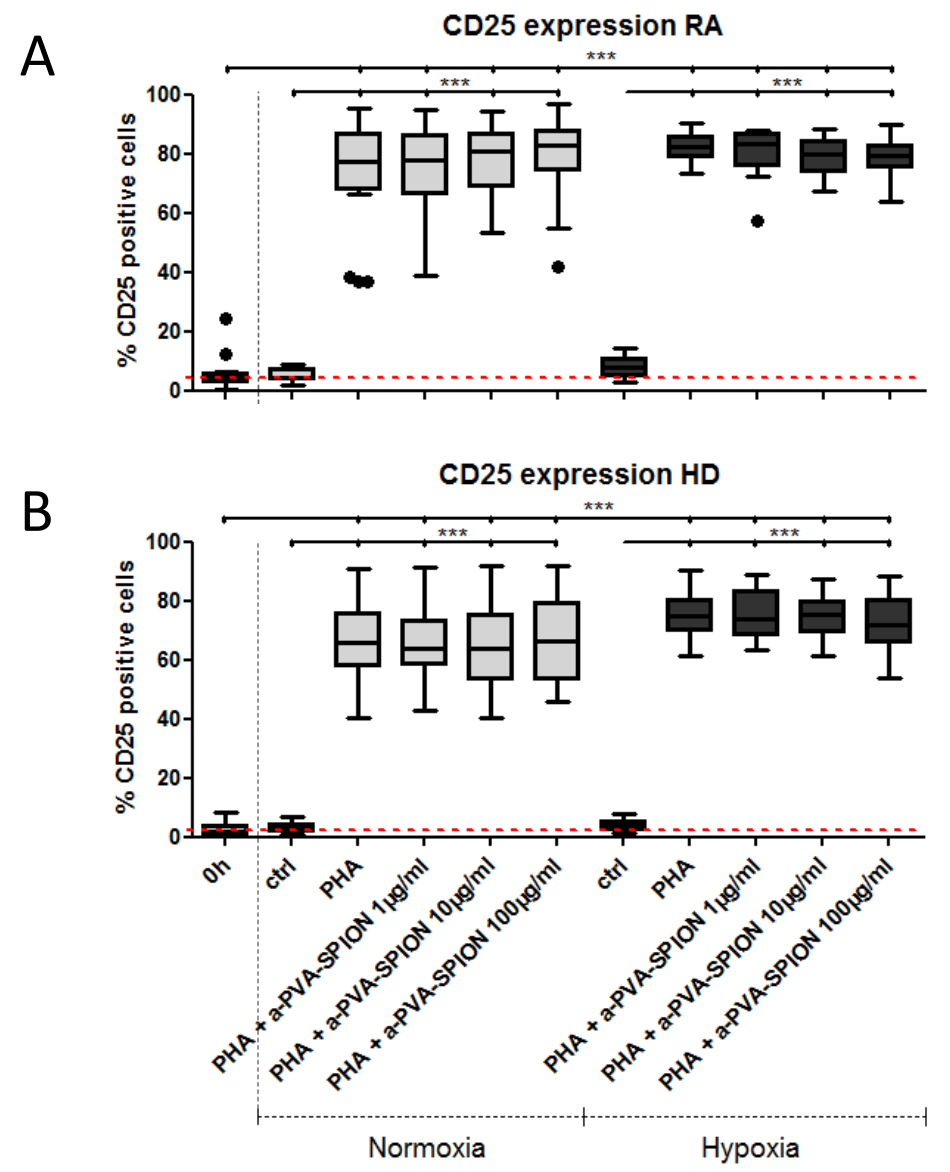
Figure 5
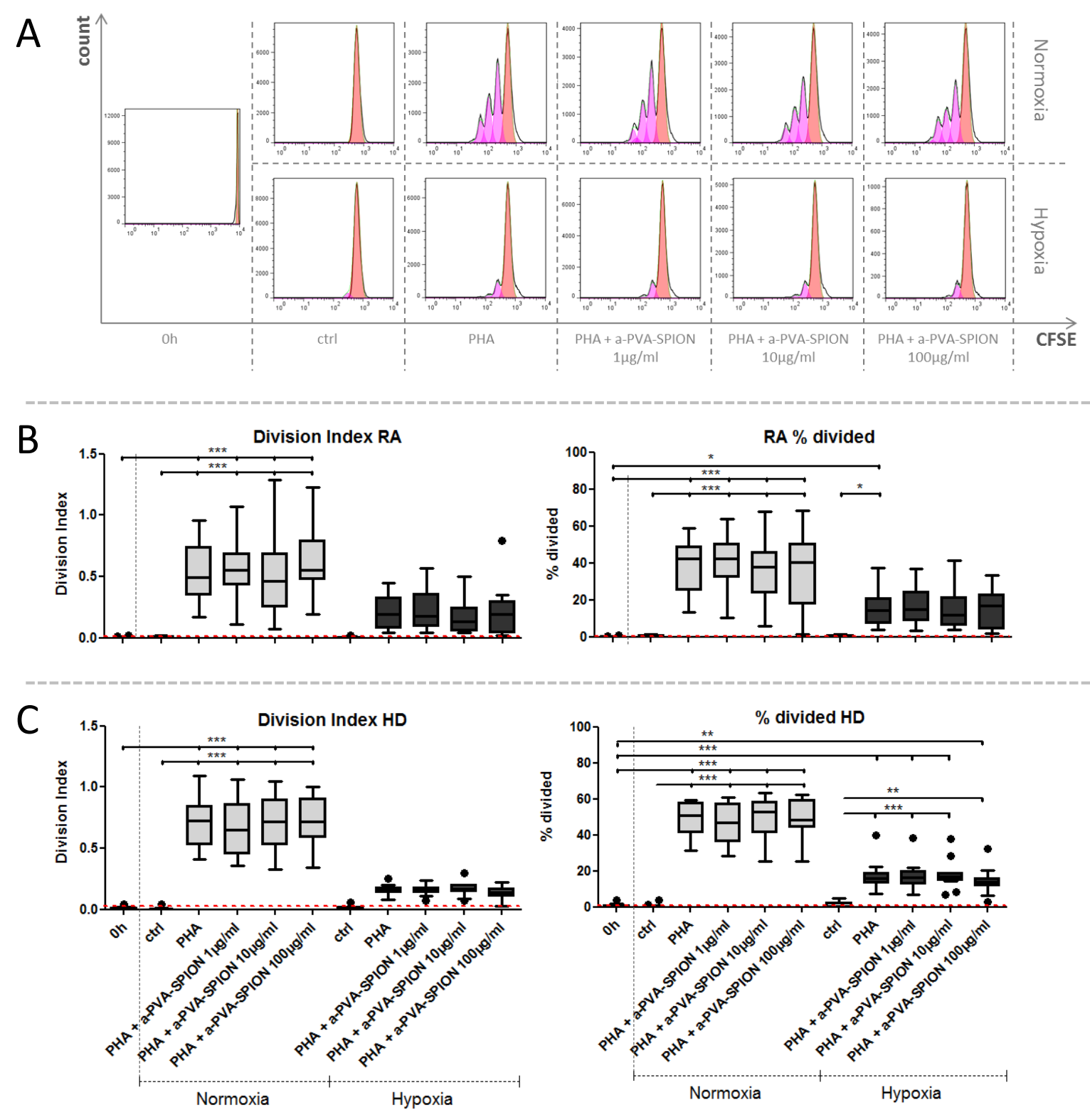